\title{
Toward Homological Characterization of Semirings by e-Injective Semimodules
}

\author{
J. Y. Abuhlail, S. N. Il’in ${ }^{\dagger}$ Y. Katsovªnd T. G. Nam ${ }^{\S}$
}

\begin{abstract}
In this paper, we introduce and study e-injective semimodules, in particular over additively idempotent semirings. We completely characterize semirings all of whose semimodules are e-injective, describe semirings all of whose projective semimodules are e-injective, and characterize onesided Noetherian rings in terms of direct sums of e-injective semimodules. Also, we give complete characterizations of bounded distributive lattices, subtractive semirings, and simple semirings, all of whose cyclic (finitely generated) semimodules are e-injective.
\end{abstract}

Key words: (e-)Injective Semimodule, (e-)Projective Semimodule, Morita Equivalence of Semirings, Simple Semirings.

MSC: 16Y60, 16D99, 06A12; 18A40, 18G05

\section{Introduction}

Semirings and semimodules, and their applications, arise in various branches of Mathematics, Computer Science, Physics, as well as many other areas of modern science (see, for instance, [14] and [13]). In the recent years, there has been a substantial amount of interest in additively idempotent semirings - among which the Boolean semifield, tropical semifields, and coordinate semirings of tropical varieties represent a set of well-known examples - originated in several extremely interesting, "nontraditional" contexts as Tropical Geometry [43], Tropical Algebra [20], $\mathbb{F}_{1}$-Geometry [8], and the Geometry of Blueprints [37], for example. Also, in the last decade, motivated by the Riemann Hypothesis [8] and tropical

${ }^{*}$ Department of Mathematics and Statistics, King Fahd University of Petroleum and Minerals, Dhahran, Saudi Arabia. Email: abuhlail@kfupm.edu.sa

${ }^{\dagger}$ Lobachevsky Institute of Mathematics and Mechanics, Kazan (Volga Region) Federal University, Kazan, Tatarstan, Russia. Email: sergey.ilyin@kpfu.ru

${ }^{\ddagger}$ Department of Mathematics, Hanover College, Hanover, IN 47243-0890, USA. Email: katsov@hanover.edu

$\S$ Institute of Mathematics, VAST, 18 Hoang Quoc Viet, Cau Giay, Hanoi, Vietnam. Email: tgnam@math.ac.vn 
varieties [43] and [9], several mathematicians have studied from different points of view semiring schemes, in particular, in [21], sheaves and homological methods on semiring schemes have been considered.

In the same time, homological characterization/classification of rings by properties of suitable classes (categories) of modules over them ( see, e.g., [35]) constitutes one of the most sustained interests and important achievements of Homological Algebra. Inspired by this, during the last three decades, a good number of important results related to this genre have been obtained in different nonabelian settings as, for example, in the homological classification of monoids 34 and distributive lattices [11]. As algebraic objects, semirings are certainly the most natural generalization of such (at first glance different) algebraic systems as rings and bounded distributive lattices, and therefore, they form an extremely interesting, natural, and important, non-abelian/non-additive setting for furthering of the homological characterization, i.e., characterizing semirings by properties of suitable classes (categories) of semimodules over them. In fact, this is an ongoing project of an substantial interest (see, e.g., [22], [24], [23], [16], [25], [17], [26], [18], [19], and [3]). In all studies regarding the homological characterization, the concepts of 'injectivity' and 'projectivity' of objects $-R$-modules, $S$-acts, $S$-semimodules, etc.- play the most leading role. Perhaps one of the most important achievements of homological algebra are due to the fact that for abelian categories of modules ${ }_{R} \mathcal{M}$ over a ring $R$ the both - universal algebra and homological algebra - approaches lead to the identical classes of modules (see, for example, [35, Sections 1.2A and 1.3A] or [12, Sect. II.6.9]). However, as we will see later on, in generally additive, but non-abelian, setting of categories of semimodules ${ }_{S} \mathcal{M}$ over a semiring $S$, these two approaches lead to two different classes of semimodules. In non-abelian (even in non-additive) settings, there have been obtained a good number of quite interesting and important results connected with the concepts of 'projectivity' and 'injectivity' based on the universal algebra approach (see, e.g., [34]). In contrast to this, in the present paper, we initiate investigations related to injective and projective semimodules defined by using the second approach, i.e., from the homological algebra point of view, heavily based on the fundamental concepts of 'extensions' and 'short exact sequences' of modules, that, in turn, lead us to the concepts of 'e-injectivity' and 'e-projectivity' of semimodules. We should mention that the latter concepts have been earlier somewise considered by some authors in semimodule settings under different terminology (see, for example, [49], 46], 47], 48], 42], [1], and [2]), but our approach, nevertheless, is slightly different and, we hope, better reflects the "homological" spirit of the matter and based on it proofs of the obtained results.

The paper is organized as follows. In Section 2, for the reader's convenience, we provide all subsequently necessary notions and facts on semirings and semimodules. 
In Section 3, we introduce the concepts of e-injectivity (-projectivity) of semimodules and establish some fundamental facts regarding them we use in a sequence. Among results of this section, we single out, in our view quite interesting and useful, Proposition 3.4 and Corollary 3.5 that provide us with a very useful tool to construct new (e-)injective semimodules from known ones.

In Section 4, we completely characterize e-injective semimodules over additively idempotent semiring having only two trivial strong one-side ideals (Proposition 4.3) and, based on this characterization, establish some fundamental facts about e-injective semimodules and their relationship with injective semimodules over additively idempotent division semirings (Theorems 4.4 and 4.5) that constitute one of the main goals and results of the paper. Also, we demonstrate (Proposition-Example 4.6) that the concepts of 'injectivity' and 'e-injectivity' for semimodules over semirings, in general, are different.

In Section 5, we characterize semirings all of whose semimodules are e-injective (Theorem 5.3), quasi-Frobenius rings in terms of projective and e-injective semimodules (Theorem 5.4), and one-sided Noetherian rings in terms of direct sums of e-injective semimodules (Theorem 5.5). These results are the 'e-injective' versions of Theorems 3.4, 3.5, and 3.6 of [17], respectively, and they also are the main results and another main goal of the paper.

Simple semirings, which are the subject of another important area of research in the theory of semirings, have quite interesting and promising applications in various fields (for example, in constructing novel semigroup actions for a potential use in public-key cryptosystems [39]). In contrast to the varieties of groups and rings, research on simple semirings started only recently, and therefore not much on the subject is known (for some recent results on simple semirings, one may consult [5], [40], [50], [27], [31], [28], [32], [33], and [30]). While complete characterizations of commutative and finite simple semirings have been given in [5] and [31], respectively, the classification of simple infinite semirings remains an important unresolved problem (see [27], [28], [32], and [33] for some recent results in this regard). In light of this and as a substantial step towards this endeavor, what constitutes one of the main goals of the paper as well, in Section 6, we give a complete description of simple semirings all of whose cyclic (finitely generated) semimodules are e-injective (Theorem 6.10). Moreover, in Section 6, we show that a bounded distributive lattice all of whose cyclic (finitely generated) semimodules are e-injective, in fact, is a finite Boolean algebra (Theorem 6.5) and, applying this result, completely characterize subtractive semirings all of whose cyclic (finitely generated) semimodules are e-injective (Theorem 6.7). Certainly, all these theorems belong to the main results of the paper.

Finally, all notions and facts of categorical algebra, used here without any comments, can be found in [38] or [7]; for notions and facts from semiring theory we refer to [14]. 


\section{Preliminaries}

2.1 Recall [14] that a semiring is a datum $(S,+, \cdot, 0,1)$ such that the following conditions are satisfied:

(1) $(S,+, 0)$ is a commutative monoid with identity element 0 ;

(2) $(S, \cdot, 1)$ is a monoid with identity element 1 ;

(3) Multiplication is distributive over addition from both sides;

(4) $0 s=0=s 0$ for all $s \in S$.

A semiring that is not a ring we call a proper semiring. A semiring $S$ is a division semiring if $(S \backslash\{0\}, \cdot, 1)$ is a group; and $S$ is a semifield if it is a commutative division semiring. Two well-known important examples of semifields are the so-called Boolean semifield $\mathbf{B}=\{0,1\}$ with $1+1=1$, and the tropical semifield $\mathbf{T}:=(\mathbb{R} \cup\{-\infty\}$, $\max ,+,-\infty, 0\})$.

As usual, a left $S$-semimodule over the semiring $S$ is a commutative monoid $\left(M,+, 0_{M}\right)$ together with a scalar multiplication $(s, m) \mapsto s m$ from $S \times M$ to $M$ which satisfies the following identities for all $s, s^{\prime} \in S$ and $m, m^{\prime} \in M$ :

(1) $\left(s s^{\prime}\right) m=s\left(s^{\prime} m\right)$;

(2) $s\left(m+m^{\prime}\right)=s m+s m^{\prime}$;

(3) $\left(s+s^{\prime}\right) m=s m+s^{\prime} m$;

(4) $1 m=m$;

(5) $s 0_{M}=0_{M}=0 m$.

Right semimodules over $S$ and homomorphisms between semimodules and semirings are defined in the standard manner. And, from now on, let $\mathcal{M}$ be the variety of commutative monoids, and $\mathcal{M}_{S}$ and ${ }_{S} \mathcal{M}$ denote the categories of right and left $S$-semimodules, respectively, over a semiring $S$.

2.2 An element $\infty \in M$ of an $S$-semimodule $M$ is infinite if $\infty+m=\infty$ for every $m \in M$; and $K \leq_{S} M$ means that $K$ is an $S$-subsemimodule of $M$. Also, we will use the following subsets of the elements of an $S$-semimodule $M$ :

$$
\begin{aligned}
& I^{+}(M):=\{m \in M \mid m+m=m\} \\
& Z(M) \quad:=\{z \in M \mid z+m=m \text { for some } m \in M\} \\
& V(M)
\end{aligned}
$$

For a semimodule $\left.M \in\right|_{S} \mathcal{M} \mid$, it is obvious that $I^{+}(M) \cap V(M)=\{0\}$, and $I^{+}(M) \leq_{S} Z(M) \leq_{S} M$.

A left $S$-semimodule $M$ is zeroic (zerosumfree, additively idempotent) if $Z(M)=$ $M\left(V(M)=0, I^{+}(M)=M\right)$. In particular, a semiring $S$ is zeroic (zerosumfree, additively idempotent) if ${ }_{S} S \in\left|{ }_{S} \mathcal{M}\right|$ is a zeroic (zerosumfree, additively idempotent) semimodule; and we say that $S$ has an infinite element if ${ }_{S} S \in\left|{ }_{S} \mathcal{M}\right|$ 
has it. For example, the Boolean semiring $\mathbf{B}=\{0,1\}$ is a commutative, zeroic, zerosumfree, additively idempotent semiring in which $\infty=1$.

2.3 A subsemimodule $A \leq{ }_{S} M$ of a semimodule $M$ is (strongly) subtractive if $\left(m+m^{\prime} \in A \Rightarrow m, m^{\prime} \in A\right) m, m+m^{\prime} \in A \Rightarrow m^{\prime} \in A$ for all $m, m^{\prime} \in M$. For each subsemimodule $A \leq{ }_{S} M$, the subsemimodule $\bar{A}:=\{m \in M \mid m+a \in A$ for some $x \in A\}$ is obviously the smallest subtractive subsemimodule of ${ }_{S} M$ containing the subsemimodule $A$, and therefore, it is called the subtractive closure of $A$; and clearly that $A$ is subtractive iff $A=\bar{A}$. A left $S$-semimodule $M$ is subtractive iff all $S$-subsemimodules of $M$ are subtractive. In particular, the semiring $S$ is left (right) subtractive iff $S$ is subtractive as a left (right) semimodule over itself. (For important properties of subtractive semirings and semimodules, the interested reader may consult [25] and [27].)

2.4 Congruences on an $S$-semimodule $M$ are defined in the standard manner, and $\operatorname{Cong}(M)$ denotes the set of all congruences on $M$. This set is non-empty since it always contains at least two congruences - the diagonal congruence $\triangle_{M}:=$ $\{(m, m) \mid m \in M\}$ and the universal congruence $M^{2}:=\{(m, n) \mid m, n \in M\}$. Any subsemimodule $L \leq{ }_{S} M$ of an $S$-semimodule $M$ induces a congruence $\equiv_{L}$ on $M$, known as the Bourne congruence, by setting $m \equiv_{L} m^{\prime}$ iff $m+l=m^{\prime}+l^{\prime}$ for some $l, l^{\prime} \in L$; and $M / L$ denotes the factor $S$-semimodule $M / \equiv_{L}$ having the canonical $S$-surjection $\pi_{L}: M \longrightarrow M / L$. Following [5], a semiring $S$ is congruence-simple iff the only congruences on $S$ are the diagonal $\Delta_{S}$ and the universal $S^{2}$; and $S$ is ideal-simple iff $S$ has exactly two ideals (namely 0 and $S)$. Note that these notions are not the same (see, e.g., [28, Examples 3.8]). Moreover, we say that a $S$ is simple iff it is simultaneously congruence-simple and ideal-simple. The classification of simple infinite semirings remains an important unresolved problem (see [27], 28], [32], and [33] for recent related results).

2.5 As usual (see, for example, [14, Chapter 17]), if $S$ is a semiring, then in the category ${ }_{S} \mathcal{M}$, a free (left) semimodule $\sum_{i \in I} S_{i}, S_{i} \cong{ }_{S} S, i \in I$, with a basis set $I$ is a direct sum (a coproduct) of $|I|$ copies of ${ }_{S} S$; a semimodule $P \in\left|{ }_{S} \mathcal{M}\right|$ is projective if it is a retract of a free semimodule. Following [22] and [24], in which there were introduced and considered in detail the tensor product bifunctors - $\otimes_{S}-: \mathcal{M}_{S} \times{ }_{S} \mathcal{M} \longrightarrow \mathcal{M}$, a semimodule $F \in{ }_{S} \mathcal{M} \mid$ is called (mono-) flat iff the functor $-\otimes_{S} F: \mathcal{M}_{S} \longrightarrow \mathcal{M}$ preserves (monomorphisms) finite limits; and the latter is equivalent to that $F$ is a filtered (directed) colimit of finitely generated free (projective) semimodules. A semimodule $\left.M \in\right|_{S} \mathcal{M} \mid$ is finitely generated (cyclic) iff $M$ is a homomorphic image of a free left $S$-semimodule with a finite basis (a homomorphic image of ${ }_{S} S$ ); a semimodule $M \in{ }_{S} \mathcal{M} \mid$ is injective if for any monomorphism $\mu: A \longmapsto B$ of left $S$-semimodules $A$ and $B$ and every homomorphism $f \in{ }_{S} \mathcal{M}(A, M)$, there exists a homomorphism $\tilde{f} \in{ }_{S} \mathcal{M}(B, M)$ such that $\tilde{f} \mu=f$. 
2.6 For the reader's convenience, we also recall some fundamental notions and facts about Morita equivalence of semirings (see, e.g., [24] and [26]) that we will use in sequence. Recall that two semirings $S$ and $T$ are said to be Morita equivalent iff the semimodule categories ${ }_{S} \mathcal{M}$ and ${ }_{T} \mathcal{M}$ are equivalent, i.e., there exist two functors $F:{ }_{S} \mathcal{M} \longrightarrow{ }_{T} \mathcal{M}$ and $G:{ }_{T} \mathcal{M} \longrightarrow{ }_{S} \mathcal{M}$ and natural isomorphisms $\eta: G F \longrightarrow I d_{S} \mathcal{M}$ and $\xi: F G \longrightarrow I d_{T} \mathcal{M}$. Following [26], a right semimodule $P_{S}$ is said to be a generator for the category $\mathcal{M}_{S}$ of right $S$-semimodules iff $S_{S}$ is a retract of a finite direct sum $\oplus_{i} P$ of $P_{S}$; and that $P_{S}$ is said to be a progenerator for $\mathcal{M}_{S}$ iff $P_{S}$ is a finitely generated projective generator. Then, by [26, Theorems 4.5 and 4.12], $F \simeq P \otimes_{S}-$ for some $(T, S)$-bisemimodule $P$ such that $P_{S}$ is a progenerator, $P^{*}:=\operatorname{Hom}_{S}\left(P_{S}, S_{S}\right)$ is a progenerator in $\mathcal{M}_{T}, T \simeq \operatorname{End}\left(P_{S}\right)$ as semirings and $G \simeq P^{*} \otimes_{T}-$.

2.7 For any left $S$-semimodule $M$, there exists the left $R$-module of differences $D(M)$ of $M$ [14, Chapter 16] (see also [29, p. 5083]) defined as the factor semimodule of the left $S$-semimodule $M \times M$ with respect to the subsemimodule $W=\{(m, m) \mid m \in M\} \subseteq M \times M$, i.e., $D(M):=(M \times M) / W$. In fact, the semimodule $D(M)$ is a left $S$-module since for any $\left(m, m^{\prime}\right) \in M \times M$ in $D(M)$ one has $\overline{\left(m, m^{\prime}\right)}+\overline{\left(m^{\prime}, m\right)}=\overline{(0,0)}$. Also, there exists the canonical $S$-homomorphism $\xi_{M}: M \longrightarrow D(M)$ given by $m \longmapsto \overline{(m, 0)}$. In the case when $M$ is a cancellative semimodule, $\xi_{M}$ is injective, and therefore, we can consider the elements $\overline{(m, 0)}$ and $m$ to be the same and any element $\overline{\left(m, m^{\prime}\right)} \in D(M)$ to be the "difference" of the elements $\overline{(m, 0)}$ and $\overline{\left(m^{\prime}, 0\right)}$, i.e., $D(M)=\left\{m-m^{\prime} \mid m, m^{\prime} \in M\right\}$. In particular, the left $S$-module of differences $D(S)$ of the regular semimodule ${ }_{S} S$ can be considered as a ring — the ring of differences of $S$ [14, Chapter 8, p. 101] with the operation of multiplication defined for all $a, b, c, d \in S$ by $\overline{(a, b)(c, d)}=$ $\overline{(a c+b d, a d+c b)}$; and if $S$ is a semiring, then the ring of differences $D(S)$ is also a semiring with the identity $\overline{(1,0)}$. Moreover, it is easy to see that $D(M)$ becomes a left $D(S)$-module with $\overline{(a, b)} \overline{\left(m_{1}, m_{2}\right)}=\overline{\left(a m_{1}+b m_{2}, a m_{2}+b m_{1}\right)}$ for all $a, b \in S$ and $m_{1}, m_{2} \in M$.

2.8 Any homomorphism $f: M \longrightarrow N$ of left $S$-semimodules induces kernel congruence $\equiv_{f}$ on $M$ such that for any $m, m^{\prime} \in M$, we have $m \equiv_{f} m^{\prime}$ iff $f(m)=$ $f\left(m^{\prime}\right)$, as well as the following subsemimodules:

$$
\begin{aligned}
& \operatorname{Ker}(f):=\{m \in M \mid f(m)=0\} \\
& \operatorname{im}(f) \quad:=\{f(m) \mid m \in M\} ; \\
& \operatorname{Im}(f):=\left\{n \in N \mid n+f(m)=f\left(m^{\prime}\right) \text { for some } m, m^{\prime} \in M\right\},
\end{aligned}
$$
called the kernel, the image, and the extended image of $f$, respectively. Notice that $\operatorname{Im}(f)=\overline{i m(f)}$ and that $\equiv{ }_{\operatorname{Ker}(f)} \subseteq \equiv_{f}$. Moreover, one can easily see that $N / i m(f)=N / \operatorname{Im}(f)$ and $M / \equiv_{f} \simeq i m(f)$. However, in general, in our nonabelian setting, as the following example shows, the $S$-semimodules $M / \operatorname{Ker}(f)$ and $i m(f)$ are not necessarily isomorphic: Indeed, the tropical semifield $\mathbf{T}:=$ 
$(\mathbb{R} \cup\{-\infty\}, \max ,+,-\infty, 0\})$ contains the Boolean semifield $\mathbf{B}$ as a subsemiring, whence $\mathbf{T}$ can be considered as a $\mathbf{B}$-semimodule in a canonical way, and let $f: \mathbf{T} \longrightarrow \mathbf{B}$ be the $\mathbf{B}$-homomorphism map given by

$$
f(x)=\left\{\begin{array}{ll}
0, & x=-\infty \\
1, & x \neq-\infty
\end{array} ;\right.
$$

Then, clearly, $f$ is surjective and $\operatorname{Ker}(f)=0$, but $\mathbf{T} / \operatorname{Ker}(f) \simeq \mathbf{T} \nsucceq \mathbf{B}=i m(f)$.

\section{3 e-Injectivity (-projectivity) of semimodules}

For the readers' convenience, briefly remind some general notions for categories with zero morphisms (see, for example, [44, Chapters 7, 8, 13]) in the context of semimodule categories ${ }_{S} \mathcal{M}$. A kernel $(K, k)$ of a homomorphism $f: A \longrightarrow B$ of left $S$-semimodules is a homomorphism $k: K \longrightarrow A$ such that (i) $f k=0$, (ii) for every homomorphism $x: X \longrightarrow A$ with $f x=0$ there exists exactly one homomorphism $i: X \longrightarrow K$ such that $x=k i$; and in this case, we write $(K, k)=\operatorname{ker} f$. By dualizing, one comes up to the concept of a cokernel $(k, K)$, $k: B \longrightarrow K$ of $f: A \longrightarrow B$, and $(k, K)=c o$ ker $f$. Then, it is obvious (or it is just readily follows from (co)completeness of semimodule categories ${ }_{S} \mathcal{M}[38$, Sections 5.1, 5.2]) that there exist ker $f$ and $c o \operatorname{ker} f$ for every homomorphism $f \in{ }_{S} \mathcal{M}(A, B):=\operatorname{Hom}_{S}(A, B)$. Furthermore, following, for example, [12, Sect. II.6.2] or [44, Sect.13.2] and without loss of generality, we can define $a$ short exact sequence in ${ }_{S} \mathcal{M}$ as a sequence

$$
0 \longrightarrow A \stackrel{f}{\longrightarrow} B \stackrel{g}{\longrightarrow} C \longrightarrow 0
$$

of semimodules $A, B, C \in\left|{ }_{S} \mathcal{M}\right|$ and homomorphisms $f$ and $g$ such that $(A, f)=$ ker $g$ and $(g, C)=c o \operatorname{ker} f$.

Let $F:{ }_{S} \mathcal{M} \longrightarrow{ }_{R} \mathcal{M}\left(G:{ }_{S} \mathcal{M} \longrightarrow{ }_{R} \mathcal{M}\right)$ be a covariant (contravariant) functor between the semimodule categories ${ }_{S} \mathcal{M}$ and ${ }_{R} \mathcal{M}$. Then, the functor $F(G)$ we say is an exact functor (or just an e-functor) if for any exact sequence $\left(^{*}\right)$ in ${ }_{S} \mathcal{M}$ the sequence

$0 \longrightarrow F(A) \stackrel{F(f)}{\longrightarrow} F(B) \stackrel{F(g)}{\longrightarrow} F(C) \longrightarrow 0(0 \longleftarrow G(A) \stackrel{G(f)}{\longleftarrow} G(B) \stackrel{G(g)}{\longleftarrow} G(C) \longleftarrow 0)$

is exact in ${ }_{R} \mathcal{M}$ as well. The following observations will prove to be useful.

Proposition 3.1 (1) A direct sum $\oplus_{i} F_{i}$ of covariant functors $F_{i}:{ }_{S} \mathcal{M} \longrightarrow{ }_{R} \mathcal{M}$, $i \in I$, is an e-functor iff each summand $F_{i}$ is an e-functor;

(2) A direct product $\prod_{i \in I} G_{i}$ of contravariant functors $G_{i}:{ }_{S} \mathcal{M} \longrightarrow{ }_{R} \mathcal{M}$, $i \in I$, is an e-functor iff each factor $G_{i}$ is an e-functor; 
(3) A retract of an e-functor is an e-functor as well.

Proof (1). It immediately follows from the observation that for an exact sequence $(*)$, the sequence

$$
0 \longrightarrow \oplus_{i} F_{i}(A) \stackrel{\oplus_{i} F_{i}(f)}{\longrightarrow} \oplus_{i} F_{i}(B) \stackrel{\oplus_{i} F_{i}(g)}{\longrightarrow} \oplus_{i} F_{i}(C) \longrightarrow 0
$$

is, in fact, a 'direct sum'

$$
0 \longrightarrow \oplus_{i}\left(F_{i}(A)\right) \stackrel{\oplus_{i}\left(F_{i}(f)\right)}{\longrightarrow} \oplus_{i}\left(F_{i}(B)\right) \stackrel{\oplus_{i}\left(F_{i}(g)\right)}{\longrightarrow} \oplus_{i}\left(F_{i}(C)\right) \longrightarrow 0
$$

of the exact sequences

$$
0 \longrightarrow F_{i}(A) \stackrel{F_{i}(f)}{\longrightarrow} F_{i}(B) \stackrel{F_{i}(g)}{\longrightarrow} F_{i}(C) \longrightarrow 0, \quad i \in I,
$$

and therefore, is an exact sequence itself.

(2). It is established in the same fashion as (1).

(3). Clearly, it is enough to consider only a "covariant" case. So, let $Q$ be a retract of an exact covariant functor $F:{ }_{S} \mathcal{M} \longrightarrow{ }_{R} \mathcal{M}$, i.e., there exist natural transformations of functors $\mu: Q \longrightarrow F$ and $\pi: F \longrightarrow Q$ such that $\pi \mu=1_{Q}$. Then, a "diagram chase" of a commutative diagram for an exact sequence $(*)$

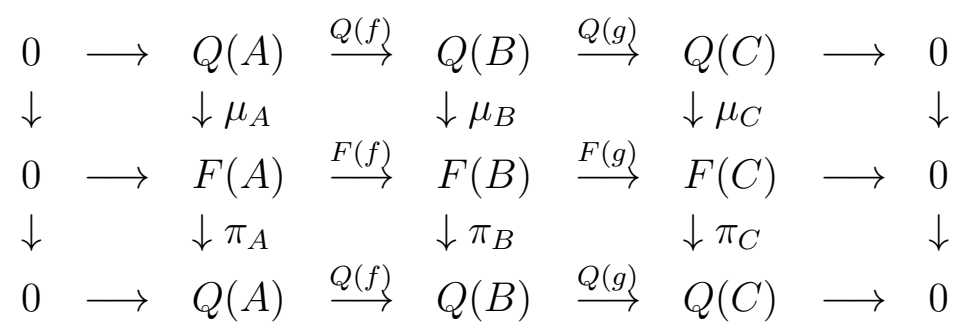

leads us to the exactness of the sequence $0 \longrightarrow Q(A) \stackrel{Q(f)}{\longrightarrow} Q(B) \stackrel{Q(g)}{\longrightarrow} Q(C) \longrightarrow 0$.

In the case when a semiring $S$ is a ring, the category ${ }_{S} \mathcal{M}$ becomes an abelian category of modules over a ring $S$. Then, there are two, at the first glance, different approaches to the concepts of injectivity and projectivity of modules: the first one is an 'universal algebra' approach presented in 2.5, and the second - a 'homological algebra' approach that in more general setting of semimodules over semirings is given by the following definition.

Definition 3.2 A semimodule $M \in\left|{ }_{S} \mathcal{M}\right|$ over a semiring $S$ is exactly-injective (exactly-projective), or shortly e-injective (e-projective), if ${ }_{S} \mathcal{M}(-, M):=$ $\operatorname{Hom}_{S}(-, M):{ }_{S} \mathcal{M} \longrightarrow \mathcal{M}\left({ }_{S} \mathcal{M}(M,-):=\operatorname{Hom}_{S}(M,-):{ }_{S} \mathcal{M} \longrightarrow \mathcal{M}\right)$ is an exact contravariant (covariant) functor.

Perhaps one of the most important achievements of homological algebra are due to the fact that for abelian categories of modules ${ }_{S} \mathcal{M}$ over a ring $S$ the both 
- universal algebra and homological algebra - approaches lead to the identical classes of modules (see, for example, [35, Sections 1.2A and 1.3A] or [12, Sect. II.6.9]). However, as we will see later on, in generally non-abelian setting of categories of semimodules ${ }_{S} \mathcal{M}$ over a semiring $S$, these two approaches lead to two different classes of semimodules. As was mentioned earlier, in non-abelian (even in non-additive) settings, there have been obtained a good number of quite interesting and important results connected with the concepts of 'projectivity' and 'injectivity' based on the universal algebra approach. In contrast to this, in the present paper, we initiate investigations related to injective and projective semimodules defined by using the second approach, i.e., from the homological algebra point of view. Thus, from Proposition 3.1 we right away obtain

Corollary 3.3 (1) A direct sum $\oplus_{i} M_{i}$ of e-projective semimodules $\left.M_{i} \in\right|_{S} \mathcal{M} \mid$, $i \in I$, is an e-projective semimodule iff each summand $M_{i}$ is an e-projective semimodule;

(2) $A$ direct product $\prod_{i \in I} M_{i}$ of e-injective semimodules $M_{i} \in\left|{ }_{S} \mathcal{M}\right|, i \in I$, is an e-injective semimodule iff each factor $M_{i}$ is an e-injective semimodule;

(3) A retract of an e-injective (e-projective) semimodule is an e-injective (eprojective) semimodule as well.

Now, let ${ }_{S} \mathcal{M}_{T}$ be a category of bisemimodules over semirings $T$ and $S$ (see, e.g., [24, Sect. 3]) and $P \in\left|{ }_{S} \mathcal{M}_{T}\right|$. Then for any semimodule $M \in\left|{ }_{S} \mathcal{M}\right|$, on the set of homomorphisms $\operatorname{Hom}_{S}\left({ }_{S} P,{ }_{S} M\right):={ }_{S} \mathcal{M}(P, M)$ there exists a natural structure of a left $T$-semimodule defined as follows: $(t f)(p)=f(p t)$ for any $f \in$ $\operatorname{Hom}_{S}\left({ }_{S} P{ }_{S} M\right), p \in P$, and $t \in T$. Moreover, there is a functor $\operatorname{Hom}_{S}\left({ }_{S} P,-\right)$ : ${ }_{S} \mathcal{M} \longrightarrow{ }_{T} \mathcal{M}$. The next result provides us with main tools for constructing examples of (e-)injective semimodules by using known (e-)injective semimodules over one semiring to produce (e-)injective semimodules over another. It includes and/or generalizes the well-known "Injective Producing Lemma" [35, Lemma 1.3.5] and [14, Proposition 17.25].

Proposition 3.4 (1) Let for a semimodule $P \in\left|{ }_{S} \mathcal{M}_{T}\right|$ the semimodule $P_{T} \in$ $\left|\mathcal{M}_{T}\right|$ be mono-flat and a semimodule $\left.M \in\right|_{S} \mathcal{M} \mid$ an injective left $S$-semimodule, then the semimodule $\left.\operatorname{Hom}_{S}\left({ }_{S} P{ }_{S} M\right) \in\right|_{T} \mathcal{M} \mid$ is an injective left T-semimodule as well;

(2) Let for a semimodule $P \in\left|{ }_{S} \mathcal{M}_{T}\right|$ the functor $P_{T} \otimes-:{ }_{T} \mathcal{M} \longrightarrow{ }_{S} \mathcal{M}$ preserve short exact sequences and a semimodule $M \in\left|{ }_{S} \mathcal{M}\right|$ an e-injective left $S$-semimodule, then the semimodule $\left.\operatorname{Hom}_{S}\left({ }_{S} P,_{S} M\right) \in\right|_{T} \mathcal{M} \mid$ is an e-injective left T-semimodule as well.

Proof (1). It is easy to see that a semimodule ${ }_{T} I \in\left|{ }_{T} \mathcal{M}\right|$ is injective iff the contravariant functor $\operatorname{Hom}_{T}\left(-{ }_{T} I\right):{ }_{T} \mathcal{M} \longrightarrow \mathcal{M}$ moves any monomorphism $\mu: A \longmapsto B$ of left $T$-semimodules $A$ and $B$ to the surjection $\operatorname{Hom}_{T}\left(\mu_{,_{T}} I\right)$ : 
$\operatorname{Hom}_{T}\left(B,_{T} I\right) \longrightarrow \operatorname{Hom}_{T}\left(A,_{T} I\right)$ in $\mathcal{M}$. Therefore, we need only to show that for any injective semimodule $M \in\left|{ }_{S} \mathcal{M}\right|$ and any monomorphism $\mu: A \longmapsto B$ in ${ }_{T} \mathcal{M}$,

$$
\begin{aligned}
\operatorname{Hom}_{T}\left(\mu, \operatorname{Hom}_{S}\left({ }_{S} P,_{S} M\right)\right): & \operatorname{Hom}_{T}\left(B, \operatorname{Hom}_{S}\left({ }_{S} P,_{S} M\right)\right) \longrightarrow \\
& \operatorname{Hom}_{T}\left(A, \operatorname{Hom}_{S}\left({ }_{S} P,_{S} M\right)\right)
\end{aligned}
$$

is a surjection in $\mathcal{M}$. However, by [24, Theorem 3.3] (or [22, Theorem 3.6]), the functor $P_{T} \otimes-:{ }_{T} \mathcal{M} \longrightarrow{ }_{S} \mathcal{M}$ is a left adjoint to the functor $\operatorname{Hom}_{S}\left({ }_{S} P,-\right)$ : ${ }_{S} \mathcal{M} \longrightarrow{ }_{T} \mathcal{M}$. Whence, from the commutative diagram

$$
\begin{aligned}
\operatorname{Hom}_{S}\left({ }_{S} P \otimes A,,_{S} M\right) \simeq & \operatorname{Hom}_{T}\left(A, \operatorname{Hom}_{S}\left({ }_{S} P,_{S} M\right)\right) \\
\downarrow & \uparrow \operatorname{Hom}_{S}\left(1_{S} P \otimes \mu, 1_{S} M\right) \\
& \uparrow \operatorname{Hom}_{T}\left(\mu, \operatorname{Hom}_{S}\left({ }_{S} P,_{S} M\right)\right) \\
\operatorname{Hom}_{S}\left({ }_{S} P \otimes B,,_{S} M\right) \simeq & \operatorname{Hom}_{T}\left(B, \operatorname{Hom}_{S}\left({ }_{S} P,_{S} M\right)\right)
\end{aligned}
$$

and the mono-flatness of the semimodule $P_{T}$, we have that $\operatorname{Hom}_{S}\left({ }_{S} P{ }_{S} M\right) \in$ $\left|{ }_{T} \mathcal{M}\right|$ is an injective semimodule.

(2). In the same fashion as it was done in (1) and assuming that $P_{T} \otimes-$ : ${ }_{T} \mathcal{M} \longrightarrow{ }_{S} \mathcal{M}$ preserves short exact sequences, one gets the e-injectivity of $\operatorname{Hom}_{S}\left({ }_{S} P,{ }_{S} M\right) \in\left|{ }_{T} \mathcal{M}\right|$.

Corollary 3.5 (cf. [35, Corollary 1.3.6B] and [14, Proposition 17.25]) Let $f$ : $S \longrightarrow T$ be a semiring homomorphism. Then the functor $\operatorname{Hom}_{S}\left({ }_{S} T,-\right):{ }_{S} \mathcal{M} \longrightarrow$ ${ }_{T} \mathcal{M}$ preserves (e-)injective semimodules.

Proof Taking into consideration that by [22, Proposition 3.8] there is the functor isomorphism $T_{T} \otimes-\simeq I d:{ }_{T} \mathcal{M} \longrightarrow \mathcal{M}$, and therefore, $T_{T} \otimes-$ is mono-flat and preserves all (co)limits as well, the statements follow right away from Proposition 3.4 .

We conclude this section with the following two remarks.

Remark 3.6 By dualization, the interested reader may easily obtain the corresponding analogs of the statements of Proposition 3.4 and Corollary 3.5 regarding (e-)projective semimodules.

Remark 3.7 It should be mentioned that the concept of e-injectivity for semimodules, in some way and using different terminology, have been earlier considered in [48, [49], [1], and [2], where the authors heavily used the obvious fact that in categories of semimodules a monomorphism $\mu: A \longmapsto B$ is a kernel of its cokernel iff the subsemimodule $A \leq B$ is a subtractive one. Moreover, it is easy to see that a semimodule $\left.{ }_{T} I \in\right|_{T} \mathcal{M} \mid$ is e-injective iff the contravariant functor $\operatorname{Hom}_{T}\left(-{ }_{T} I\right):{ }_{T} \mathcal{M} \longrightarrow \mathcal{M}$ moves any monomorphism $\mu: A \longmapsto B$ with $\mu(A)$ being a subtractive subsemimodule of a left $T$-semimodule $B$ to the surjection $\operatorname{Hom}_{T}\left(\mu,_{T} I\right): \operatorname{Hom}_{T}\left(B,_{T} I\right) \longrightarrow \operatorname{Hom}_{T}\left(A,_{T} I\right)$ in $\mathcal{M}$ and $\left(\operatorname{Hom}_{T}\left(\mu,_{T} I\right)\right.$, $\left.\operatorname{Hom}_{T}\left(A,_{T} I\right)\right)=\operatorname{coker}\left(\operatorname{Hom}_{T}\left(\pi,_{T} I\right)\right)$, where $\pi: B \longrightarrow B / \mu(A)$ is the natural 
surjection; in other words, $\left.{ }_{T} I \in\right|_{T} \mathcal{M} \mid$ is e-injective iff, for any $T$-homomorphism $\varphi: A \longrightarrow I$, there exists a $T$-homomorphism $\psi: B \longrightarrow I$ such that $\varphi=\psi \mu$ and, for any $T$-homomorphisms $\psi_{1}, \psi_{2}: B \longrightarrow I$, the equality $\psi_{1} \mu=\psi_{2} \mu$ implies $\psi_{1}+\chi_{1}=\psi_{2}+\chi_{2}$ for suitable $\chi_{1}$ and $\chi_{2}$ for which $\chi_{1} \mu=0=\chi_{2} \mu$. And for that reason, the terminology used in those works was certainly influenced by these facts. Our approach and "ideology" are slightly different.

\section{4 e-Injective semimodules over additively idem- potent semirings}

In this section, we consider the structure of e-injective semimodules as well as relations between e-injective and injective semimodules over additively idempotent semirings. To do so, we need first to establish some useful facts.

Proposition 4.1 Let $S$ be a zerosumfree semiring and $M \in\left|{ }_{S} \mathcal{M}\right|$ an e-injective $S$-semimodule. Then there exists an element $z \in M$ such that $m+z=z$ for all $m \in M$, and in addition, $s z=z$ for every $s \in S \backslash\{0\}$ if $S$ is an entire semiring.

Proof For an e-injective semimodule $\left.M \in\right|_{S} \mathcal{M} \mid$ consider the relation $\sim$ on the left $S$-semimodule $S \times M \in{ }_{S} \mathcal{M} \mid$ defined as follows: $\left(s_{1}, m_{1}\right) \sim\left(s_{2}, m_{2}\right)$ iff $s_{1}=s_{2}$ and there are $m_{i}^{\prime}, m_{i}^{\prime \prime} \in M, x_{i}, x_{i}^{\prime} \in S(i=1,2, \ldots, n)$ such that $x_{i}+x_{i}^{\prime}=s_{1}$ and $m_{1}+\sum_{i=1}^{n} x_{i} m_{i}^{\prime}=m_{2}+\sum_{i=1}^{n} x_{i} m_{i}^{\prime \prime}$ for some natural number $n$. As was shown in [17, Propositions 1.6 and 1.7], $\sim$ is an $S$-congruence on $S \times M \in$ ${ }_{S} \mathcal{M} \mid$ and there exists an $S$-monomorphism $\alpha: M \longrightarrow S \times M / \sim, m \mapsto\left[0_{S}, m\right]$ such that $\left[0_{S}, m\right]+\left[1,0_{M}\right]=\left[1,0_{M}\right]$ for all $m \in M$, respectively. Obviously, $\alpha(M)$ is a subtractive subsemimodule of the semimodule $S \times M / \sim$. Therefore as well as taking into considertation Remark 3.7, there exists an $S$-homomorphism $\beta: S \times M / \sim \longrightarrow M$ such that $\beta \alpha=i d_{M}$. Let $z:=\beta\left(\left[1,0_{M}\right]\right)$; and as it was shown in [17, Proposition 1.7], $m+z=z$ for all $m \in M$.

If in addition, $S$ is an entire semiring, it is easy to see that for the natural embedding $\mu: M \longmapsto \operatorname{Ext}(M)$, where $\operatorname{Ext}(M):=M \cup\{\infty\}$ with $0 \infty=0$ and $s \infty=\infty$ for all $s \in S \backslash\{0\}$ and $m+\infty=\infty$ for all $m \in \operatorname{Ext}(M)$, there is a subtractive semimodule $\mu(M) \leq \operatorname{Ext}(M)$. Since ${ }_{S} M$ is e-injective, by Remark 3.7, there exists $\gamma: \operatorname{Ext}(M) \longrightarrow M$ such that $\gamma \mu=i d_{M}$, and clearly $z=\gamma(\infty)$ and $s z=s \gamma(\infty)=\gamma(s \infty)=\gamma(\infty)=z$ for every $s \in S \backslash\{0\}$.

Lemma 4.2 If a zerosumfree semiring $S$ has only two trivial strongly subtractive left (right) ideals, then $S$ is entire.

Proof Indeed, suppose that $a b=0$ for some $a, b \in S$ and $b \neq 0$. Since $S$ is zerosumfree, the left ideal

$$
\left(0:{ }_{S} b\right):=\{s \in S \mid s b=0\}
$$


is strongly subtractive and $1 \notin\left(0:{ }_{S} b\right)$. Hence, $\left(0:{ }_{S} b\right)=0$ and $a=0$.

The next observation, complementing Proposition 4.1, completely describes einjective semimodules over additively idempotent semirings with only two trivial one-sided strongly subtractive ideals.

Proposition 4.3 A left $S$-semimodule $\left.M \in\right|_{S} \mathcal{M} \mid$ over an additively idempotent semiring $S$ with only two trivial left strongly subtractive ideals is e-injective iff it possesses the infinite element $\infty \in M$ such that $s \infty=\infty$ for all $s \in S \backslash\{0\}$.

Proof $\Longrightarrow$. By Lemma 4.2,S is an additively idempotent, zerosumfree, and entire, semiring. Whence, the result follows from Proposition 4.1.

$\Longleftarrow$. Let $\mu: A \longmapsto B$ be the inclusion map from a subtractive subsemimodule ${ }_{S} A$ to a semimodule ${ }_{S} B$ in ${ }_{S} \mathcal{M}$. For $S$ is additively idempotent, the additive reducts of all $S$-semimodules are idempotent monoids as well. Moreover, ${ }_{S} A$ is a strongly subtractive subsemimodule of ${ }_{S} B$ : Indeed, if $x+y \in A$ for some $x, y \in B$, then $x+(x+y)=x+y \in A$ and $y+(x+y)=x+y \in A$, and hence, $x, y \in A$.

Next, for an $S$-semimodule homomorphism $f: A \longrightarrow M$, define a map $g$ : $B \longrightarrow M$ as follows:

$$
g(b)=\left\{\begin{array}{ll}
f(b), & b \in A \\
\infty, & b \notin A
\end{array} .\right.
$$

It is obvious that $f=g \mu$, and we claim that actually $g$ is an $S$-semimodule homomorphism, too.

Indeed, for any $b, c \in B$, if $b+c \in A$, then $b, c \in A$ since $A$ is a strongly subtractive subsemimodule of $B$, and therefore, $g(b+c)=f(b+c)=f(b)+f(c)=$ $g(b)+g(c)$; if $b+c \notin A$, then we have the following two cases to consider:

Case 1: $b \in A$ and $c \notin A$. Then, $g(b+c)=\infty=f(b)+\infty=g(b)+g(c)$;

Case 2: $b \notin A$ and $c \notin A$. Then, $g(b+c)=\infty=\infty+\infty=g(b)+g(c)$.

Now let $b \in B$ and $s \in S$. For $A \leq_{S} B$ is a strongly subtractive subsemimodule, the left ideal

$$
I_{b}:=\{s \in S \mid s b \in A\}
$$

is a strongly subtractive left ideal in $S$. Hence, $I_{b}=0$ or $I_{b}=S$; and $s b \in A$ iff $s=0$ or $b \in A$, or equivalently, $s b \notin A$ iff $s \neq 0$ and $b \notin A$. Furthermore, we always have $s \infty=\infty$ for all $s \in S \backslash\{0\}$. From these observations, we immediately get $g(s b)=s g(b)$, and therefore, $g$ is an $S$-semimodule homorphism.

Finally, let $\pi: B \longrightarrow B / A$ be the natural surjection. It is easy to see that there is a homomorphism $\gamma \in \operatorname{Hom}_{S}(B / A, M)$ such that the image $\gamma([b])$ of any non-zero element $[b] \in B / A$ is the infinite element $\infty \in M$. Moreover, it is quite clear that for any $\alpha, \alpha^{\prime} \in \operatorname{Hom}_{S}(B, M)$ such that $\alpha \mu=\alpha^{\prime} \mu$, we have $\alpha+\gamma \pi=\alpha^{\prime}+\gamma \pi$ with $\gamma \pi \mu=0$. Hence, by Remark 3.7, we get $\left(\operatorname{Hom}_{S}(\mu, M), \operatorname{Hom}_{S}(A, M)\right)=c o \operatorname{ker}\left(\operatorname{Hom}_{S}(\pi, M)\right)$, and ${ }_{S} M$ is an e-injective $S$-semimodule. 
It is obvious that every division semiring has only two trivial strongly left/right subtractive ideals. So, as a consequence of Proposition 4.3, among other, in our view, interesting and important observations, in the next theorem we obtain a complete characterization of e-injective semimodules over additively idempotent division semirings.

Theorem 4.4 For an additively idempotent division semiring $D$ the following statements are true:

(1) A left D-semimodule $M$ is e-injective iff $M$ has an infinite element;

(2) Every left D-semimodule can be embedded in an e-injective D-semimodule;

(3) Every injective left D-semimodule is e-injective;

(4) Every finite left D-semimodule is e-injective;

(5) The regular $D$-semimodule ${ }_{D} D$ (and therefore, every finitely generated left $D$-semimodule) is e-injective iff $D \simeq \mathbf{B}$.

Proof So, let $D$ be an additively idempotent, and therefore, zerosumfree, division semiring.

$(1) \Longrightarrow$. This follows immediately from Proposition 4.3.

$\Longleftarrow$. Let $M$ be a left $D$-semimodule with the infinite element $\infty$. For every $s \in D \backslash\{0\}$, we have $s \infty+\infty=\infty$, and hence, $\infty=\infty+s^{-1} \infty=s^{-1} \infty$, whence $s \infty=\infty$. From this observation and Proposition 4.3, we conclude that ${ }_{D} M$ is e-injective.

(2) For every left $D$-semimodule $M$, the left $D$-semimodule $\operatorname{Ext}(M):=M \cup$ $\{\infty\}$ has an infinite element, and therefore by (1), it is e-injective.

(3) Let $M$ be an injective left $D$-semimodule. By [17, Proposition 1.7], $M$ has an infinite element $\infty$, whence $M$ is e-injective by (1).

(4) Let $M=\left\{m_{1}, \cdots, m_{n}\right\}$. Then, $\infty:=\sum_{i=1}^{n} m_{i}$ is an infinite element of $M$ and by $(1)_{D} M$ is e-injective.

$(5) \Longrightarrow$. Assume that ${ }_{D} D$ is e-injective. Since $D$ is zerosumfree and by Proposition 4.1, it has an infinite element $\infty$. It follows that $\infty=\infty+\infty^{2}=$ $(\infty+1) \infty=\infty^{2}$, and hence, $1=\infty$. Then, for every $d \in D \backslash\{0\}$, we have $d^{-1}+1=1$ and $1=d+1=d\left(1+d^{-1}\right)=d \cdot 1=d$. Thus, $D \simeq \mathbf{B}$.

$\Longleftarrow$. This is clear from (1) and (4).

Our next observation extends statements (2) and (3) of Theorem 4.4 to arbitrary idempotent semirings and actually establishes the e-injective completeness of categories of $S$-semimodules ${ }_{S} \mathcal{M}$ over additively idempotent semirings $S$ (the injective completeness of those categories has been established earlier in [22, Theorem 4.2]), namely:

Theorem 4.5 For an additively idempotent semiring $S$ the following statements are true:

(1) Every left S-semimodule can be embedded in an e-injective S-semimodule; 
(2) Every injective left S-semimodule is e-injective.

Proof (1) Since $S$ is additively idempotent, there is the obvious semiring embedding $\mu: \mathbf{B} \longmapsto S$ of the Boolean semifield $\mathbf{B}$ into a subsemiring $S$. Therefore, any semimodule $M \in{ }_{S} \mathcal{M} \mid$ has the canonical B-semimodule structure, i.e., $M \in$ $\left.\right|_{\mathbf{B}} \mathcal{M} \mid$. By (2) of Theorem 4.4, there exists an an e-injective B-semimodule $Q$ such that $M \leq{ }_{\mathrm{B}} Q$. Then, taking into consideration obvious embeddings

$$
{ }_{S} M \simeq \operatorname{Hom}_{S}(S, M) \leq \operatorname{Hom}_{\mathbf{B}}(S, M) \leq \operatorname{Hom}_{\mathbf{B}}(S, Q),
$$

and applying Corollary 3.5, we have that $\operatorname{Hom}_{\mathbf{B}}\left({ }_{S} S, Q\right)$ is an e-injective left $S$ semimodule.

(2) Let $\left.M \in\right|_{S} \mathcal{M} \mid$ be an injective left $S$-semimodule. By (1), in the category ${ }_{S} \mathcal{M}$ there exists an embedding $\mu: M \longmapsto Q$, where $Q$ is an e-injective $S$-semimodule. Whence, as ${ }_{S} M$ is an injective $S$-semimodule, there exists an $S$-homomorphism $\pi: Q \longrightarrow M$ such that $\pi \mu=i d_{M}$, i.e., ${ }_{S} M$ is a retract of ${ }_{S} Q$; and by (3) of Corollary 3.3, we end the proof.

Theorems 4.4 and 4.5 show that in the category ${ }_{S} \mathcal{M}$ over an additively idempotent semiring $S$, the class of injective semimodules, $\operatorname{Inj}\left({ }_{S} \mathcal{M}\right)$, is a subclass of the class of e-injective semimodules, $e-\operatorname{Inj}\left({ }_{S} \mathcal{M}\right)$. In fact, our next observation shows that for an additively idempotent division semiring $S$, the subclass $\operatorname{Inj}\left({ }_{S} \mathcal{M}\right)$ is always a proper one of the class $e-\operatorname{Inj}\left({ }_{S} \mathcal{M}\right)$, namely:

Proposition-Example 4.6 Let $D$ be an additively idempotent division semiring. Then $\operatorname{Inj}\left({ }_{D} \mathcal{M}\right) \subset e-\operatorname{Inj}\left({ }_{D} \mathcal{M}\right)$.

Proof For $D$ is a zerosumfree division semiring, there exists a surjective semiring homomorphism $\pi: D \longrightarrow$ B. By [26, Lemma 5.2], the restriction functor $\pi^{\#}$ : ${ }_{\mathbf{B}} \mathcal{M} \longrightarrow{ }_{D} \mathcal{M}$ preserves non-injective semimodules. Let $M \in{ }_{\mathbf{B}} \mathcal{M} \mid$ be a nondistributive finite lattice, then ${ }_{\mathbf{B}} M$ is non-injective $\mathbf{B}$-semimodule [10, Theorem 4], and therefore, $\pi^{\#}(M) \in\left|{ }_{D} \mathcal{M}\right|$ is non-injective $D$-semimodule. However, by (4) of Theorem 4.4, $\pi^{\#}(M) \in e-\operatorname{Inj}\left({ }_{D} \mathcal{M}\right)$.

In light of the observations above, we finish this section by posting the following, in our view interesting and perspective, conjecture and problems.

Conjecture $1 \operatorname{Inj}\left({ }_{S} \mathcal{M}\right)=e-\operatorname{Inj}\left({ }_{S} \mathcal{M}\right)$ iff a semiring $S$ is a ring.

Problem 2 Is (1) of Theorem 4.5 true for all additively regular semirings $S$ ?

Problem 3 Does an e-injective envelope exist for every semimodule $M \in\left|{ }_{S} \mathcal{M}\right|$ over an additively idempotent semiring $S$ ?

Problem 4 Describe all semirings $S$ such that $\operatorname{Inj}\left({ }_{S} \mathcal{M}\right) \subset e-\operatorname{Inj}\left({ }_{S} \mathcal{M}\right)$. 


\section{Characterizations of some special classes of semirings}

In [17], there were obtained semiring analogs of well-known characterizations of (classical) semisimple, quasi-Frobenius, and one-sided Noetherian rings by means of injective semimodules over them. Motivated by this, it is quite natural to consider characterizations of those classes of semirings in terms of e-injective semimodules over them. And therefore, establishing the 'e-injective' versions of those characterizations constitutes the main goal of this section.

Proposition 5.1 (cf. [17, Theorem 1.2]) Let $S$ be a zerosumfree semiring and $\left\{M_{i}\right\}_{i \in I}$ a family of left nonzero $S$-semimodules. Then the left $S$-semimodule $\oplus_{i \in I} M_{i}$ is e-injective iff all semimodules $\left\{M_{i}\right\}_{i \in I}$ are e-injective and $|I|<\infty$.

Proof $\Longrightarrow$. Let $M=\oplus_{i \in I} M_{i}$ be an e-injective left $S$-semimodule. By Corollary 3.3 (3), for every $i \in I$, the $S$-semimodule $M_{i}$ is e-injective, and hence, by Proposition 4.1, there exists the element $z_{i} \in M_{i}$ such that $m_{i}+z_{i}=z_{i}$ for all $m_{i} \in M_{i}$. Then, the image of the canonical embedding $\mu: M \succ \bar{M}:=\prod_{i \in I} M_{i}$ is clearly a subtractive subsemimodule, and therefore, there exists a homomorphism $\varphi: \bar{M} \longrightarrow M$ such that $\varphi \mu=1_{M}$.

Now let $z:=\left(z_{i}\right)_{i \in I} \in \bar{M}$. Since $\varphi(z) \in M$, there exists a finite subset $J \subseteq I$ and a collection of elements $\left(m_{j}\right)_{j \in J}$ with $m_{j} \in M_{j}$, such that $\varphi(z)=\Sigma_{j \in J} m_{j}$. We shall show that actually $J=I$. Indeed, for any index $i \in I \backslash J$, it is easy to see that $\mu\left(z_{i}\right)+z=z$ and

$$
\Sigma_{j \in J} m_{j}=\varphi(z)=\varphi\left(\mu\left(z_{i}\right)+z\right)=\varphi\left(\mu\left(z_{i}\right)\right)+\varphi(z)=z_{i}+\Sigma_{j \in J} m_{k} ;
$$

and as $i \notin J$, one has that $z_{i}=0$. Whence, for any $m_{i} \in M_{i}$, we have that $0=z_{i}=z_{i}+m_{i}=0+m_{i}=m_{i}$, i.e., $M_{i}=0$ what contradicts to $M_{i} \neq 0$. Thus, $J=I$.

$\Longleftarrow$. It follows straightforwardly from Corollary 3.3.

For given a semiring congruence $\theta$ on a semiring $S$ and a semimodule $M \in$ $\left|{ }_{S} \mathcal{M}\right|$, we say that an element $m \in M$ is compatible with $\theta$ iff $s_{1} \theta s_{2} \Longrightarrow s_{1} m=$ $s_{2} m$ for all $s_{1}, s_{2} \in S$; and let $M(\theta):=\{m \in M \mid m$ is compatible with $\theta\}$. As was shown in [17, Propositions 1.2 and 1.3], $M(\theta)$ is a left $S / \theta$-semimodule and $M(\theta) \simeq \operatorname{Hom}_{S}(S / \theta, M)$ as left $S$-semimodules. From these observations and Corollary 3.5, we immediately obtain the following e-injective analog of [17, Proposition 1.4]:

Proposition 5.2 If $M$ is an e-injective left $S$-semimodule, then $M(\theta)$ is an e-injective left $S / \theta$-semimodule.

In the next result, we complement [17, Theorem 3.4] and describe the class of semirings all of whose semimodules are e-injective. 
Theorem 5.3 The following conditions for a semiring $S$ are equivalent:

(1) All left $S$-semimodules are e-injective;

(2) $S$ is a classical semisimple ring.

Proof $(1) \Longrightarrow(2)$. By our hypothesis, $S^{(\mathbb{N})}$ is an e-injective left $S$-semimodule. Let $\theta$ be the Bourne relation on $S$ corresponding to the ideal $V(S)$ (see 2.2 and 2.4). Then, it is easy to see that $S / \theta$ is a zerosumfree semiring, and for the einjective left $S$-semimodule $S^{(\mathbb{N})}$ there is the natural $S / \theta$-semimodule isomorphism $S^{(\mathbb{N})}(\theta) \simeq S(\theta)^{(\mathbb{N})}$, and therefore, by Proposition 5.2, $S^{(\mathbb{N})}(\theta)$ is an e-injective left $S / \theta$-semimodule. From the latter and Proposition 5.1, $S(\theta)=0$.

Also, it is easy to verify that the image of the canonical embedding $\xi_{V(S)}$ : $V(S) \longmapsto D(S)$ (see 2.7) is a subtractive $S$-subsemimodule. For ${ }_{S} S$ is e-injective, the natural injection $\mu: V(S) \longmapsto S$ can be extended to an $S$-homomorphism $\psi: D(S) \longrightarrow S$ such that $\psi \xi_{V(S)}=\mu$. Since $D(S)$ is a ring, it is clear that $e:=\psi\left(1_{D(S)}\right) \in V(S)$. For every $s \in V(S)$, we have $s=\mu(s)=\psi\left(\xi_{V(S)}(s)\right)=$ $\psi\left(s 1_{D(S)}\right)=s \psi\left(1_{D(S)}\right)=s e$. In particular, $e^{2}=e$, and so $s(1-e)=s e(1-$ $e)=s(e-e)=s 0=0$ for all $s \in V(S)$, and applying [17, Lemma 1.1], we conclude that $1=e \in V(S)$, i.e., $S$ is actually a ring and, by [36, Theorem 1.2.9], even a semisimple ring as the concepts of injectivity and e-injectivity coincide for modules over rings.

$(2) \Longrightarrow(1)$. This follows immediately from [36, Theorem 1.2.9] as the concepts of injectivity and e-injectivity coincide for modules over rings.

The celebrated Faith-Walker Theorem provides the characterization of quasiFrobenius rings as rings over which the classes of projective and injective modules coincide (e.g., [4, Theorem 31.9], or [35, Theorem 15.9]). In [17, Theorem 3.5], this characterization has been generalized in the semiring setting, and our next result is an 'e-injective' version of [17, Theorem 3.5].

Theorem 5.4 The following conditions for a semiring $S$ are equivalent:

(1) $S$ is a quasi-Frobenius ring;

(2) All projective left $S$-semimodules are e-injective;

(3) All e-injective left $S$-semimodules are projective, and the $S$-semimodule $S / V(S)$ can be embedded in an e-injective left $S$-semimodule.

Proof $(1) \Longrightarrow(2)$. This follows from [4, Theorem 31.9], or [35, Theorem 15.9], and the fact that the notions of injectivity and e-injectivity coincide for modules over rings.

$(2) \Longrightarrow(1)$. Since the projective left $S$-semimodule $S^{(\mathbb{N})}$ is e-injective, as in the proof of the implication $(1) \Longrightarrow(2)$ of Theorem 5.4 we have that $S$ is a ring and then use [4, Theorem 31.9].

$(1) \Longrightarrow(3)$. If $S$ is a ring, $S / V(S)=0$ is an e-injective $S$-semimodule. Therefore, the statement follows from [4, Theorem 31.9]. 
$(3) \Longrightarrow(1)$. Let $\theta$ be the Bourne relation on $S$ induced by $V(S)$. By the hypothesis, $S / \theta$ can be embedded in an e-injective left $S$-semimodule $M$. It is easy to see that every element of $S / \theta$ is compatible with $\theta$, and hence, $S / \theta$ can be embedded in $M(\theta)$. Let $I$ be an infinite set such that $|I| \geq|S|$. By Corollary $3.3(2), M^{I}$ is an e-injective left $S$-semimodule, and hence, a projective left $S$ semimodule; and therefore, by [17, Theorem 2.1], $(M,+, 0)$ is an abelian group. For ${ }_{S} M$ is e-injective, by Proposition $5.2, M(\theta)$ is an e-injective left semimodule over the zerosumfree semiring $S / \theta$; and by Proposition 4.1, there exists an element $z \in M(\theta)$ such that $z+m=z$ for all $m \in M(\theta)$. In particular, $z+z=z$, and, hence, as $M(\theta) \subseteq M$ and $(M,+, 0)$ is a group, $z=0$, and therefore, $M(\theta)=0$ and $S / \theta=0$, i.e. $S$ is a ring. From the latter, the implication follows from [4, Theorem 31.9].

Also, the famous and very important characterization of Noetherian rings as rings over which direct sum of injective modules is always an injective module given by H. Bass and Z. Papp (see, e.g., [35, Theorem 3.46]) has been generalized in the semiring setting in [17, Theorem 3.6], and we conclude this section by an 'e-injective' version of the latter.

Theorem 5.5 The following conditions for a semiring $S$ are equivalent:

(1) $S$ is a left Noetherian ring;

(2) Every direct sum of e-injective left $S$-semimodules is e-injective, and the $S$-semimodule $S / V(S)$ can be embedded in an e-injective left $S$-semimodule.

Proof $(1) \Longrightarrow(2) . S / V(S)$ is obviously the zero e-injective left $S$-semimodule for any left Noetherian ring $S$, and the implication follows from [35, Theorem 3.46].

$(2) \Longrightarrow(1)$. Let $\theta$ be the Bourne relation on $S$ induced by $V(S)$. By the hypothesis, $S / \theta$ can be embedded in an e-injective left $S$-semimodule $M$. It is easy to see that every element of $S / \theta$ is compatible with $\theta$, and hence, $S / \theta$ can be embedded in $M(\theta)$. Then, as $M^{(\mathbb{N})}$ is an e-injective left $S$-semimodule and applying Proposition 5.2, we have that $M(\theta)^{(\mathbb{N})} \simeq M^{(\mathbb{N})}(\theta)$ is an e-injective left $S / \theta$-semimodule. For $S / \theta$ is zerosumfree and applying Proposition 5.1, we have $M(\theta)=0$ and, hence, $S / \theta=0$. Therefore, by [35, Theorem 3.46], $S$ is a left Noetherian ring.

\section{Semirings all of whose finitely generated semi- modules are e-injective}

As was shown in [19, Corollary 3.2], every semimodule can be represented, in a canonical way, as a colimit of its cyclic subsemimodules. This observation motivates studying of semirings over which any semimodule is a colimit of cyclic 
semimodules possessing some special properties (see, e.g., [3], [19]). Thus, it is quite natural that the main goal of this section is to present complete characterizations of semirings, belonging to some important special classes of semirings, all of whose cyclic (finitely generated) semimodules are e-injective. But first we make some interesting and useful general observations.

Proposition 6.1 The class of semirings all of whose cyclic (finitely generated) left semimodules are e-injective is closed under homomorphic images and finite products.

Proof Let $\mathfrak{C}(\mathfrak{F})$ be the class of semirings all of whose cyclic (finitely generated) left semimodules are e-injective. First consider the statement for the class $\mathfrak{C}$. So, let $S, T$ be semirings with $S \in \mathfrak{C}$ and $\pi: S \longrightarrow T$ a surjective semiring homomorphism. We claim that $T \in \mathfrak{C}$. By [24, Section 4], $\pi$ induces two functors - the restriction of scalars functor $\pi^{\#}:{ }_{T} \mathcal{M} \longrightarrow{ }_{S} \mathcal{M}$ and the extension functor $\pi_{\#}:=T \otimes_{S}-:{ }_{S} \mathcal{M} \longrightarrow{ }_{T} \mathcal{M}$ - such that $\pi_{\#}$ is a left adjoint to the functor $\pi^{\#}$, i.e., $\pi_{\#} \dashv \pi^{\#}$ [24, Proposition 4.1]. Moreover, by [24, Proposition 4.6], $\pi_{\#} \pi^{\#} \simeq$ $I d_{T} \mathcal{M}$; also, it is easy to see that a short sequence $0 \longrightarrow A \stackrel{f}{\longrightarrow} B \stackrel{g}{\longrightarrow} C \longrightarrow 0$ is exact in ${ }_{T} \mathcal{M}$ iff it is exact in ${ }_{S} \mathcal{M}$, and any semimodule $\left.M \in\right|_{T} \mathcal{M} \mid$ is cyclic iff $M \in\left|{ }_{S} \mathcal{M}\right|$ is cyclic. By using these observations and the assumption $S \in \mathfrak{C}$, it is easy to see that the functor $\operatorname{Hom}_{T}(-, M):{ }_{T} \mathcal{M} \longrightarrow \mathcal{M}$ preserves short exact sequences iff the functor $\operatorname{Hom}_{S}(-, M):{ }_{S} \mathcal{M} \longrightarrow \mathcal{M}$ does it, and therefore, $T \in \mathfrak{C}$, i.e., $\mathfrak{C}$ is closed under homomorphic images. Using the same arguments, we obtain the closedness of the class $\mathfrak{F}$ with respect of homomorphic images.

It is clear that it is enough to show only for two semirings $S_{1}, S_{2} \in \mathfrak{C}(\mathfrak{F})$ that $S:=S_{1} \oplus S_{2} \in \mathfrak{C}(\mathfrak{F})$ as well. Obviously, any $S$-semimodule $M \in{ }_{S} \mathcal{M}$ actually is the direct sum of its $S$-subsemimodules $S_{1} M:=\left\{\left(s_{1}, 0\right) M \mid s_{1} \in S_{1}\right\}$ and $S_{2} M:=\left\{\left(0, s_{2}\right) M \mid s_{2} \in S_{2}\right\}$, i.e., $M=S_{1} M \oplus S_{2} M$. Moreover, it is easy to see that any homomorphism $f: M=S_{1} M \oplus S_{2} M \longrightarrow S_{1} N \oplus S_{2} N=N$ between $S$ semimodules $M, N \in{ }_{S} \mathcal{M}$ is, in fact, the direct sum of the two corresponding $S_{1^{-}}$ homomorphism $f_{1}: S_{1} M \longrightarrow S_{1} N$ and $S_{2}$-homomorphism $f_{2}: S_{2} M \longrightarrow S_{2} N$ between $S_{1} M, S_{1} N \in{ }_{S_{1}} \mathcal{M}$ and each $S_{2} M, S_{2} N \in{ }_{S_{2}} \mathcal{M}$, respectively. From observations for any functor $\operatorname{Hom}_{S}(-, M):{ }_{S} \mathcal{M} \longrightarrow \mathcal{M}$, we readily have $\operatorname{Hom}_{S}(-, M)=$ $\operatorname{Hom}_{S}\left(-, S_{1} M \oplus S_{2} M\right) \simeq \operatorname{Hom}_{S}\left(-, S_{1} M\right) \times \operatorname{Hom}_{S}\left(-, S_{2} M\right) \simeq \operatorname{Hom}_{S_{1}}\left(-, S_{1} M\right) \times$ $\operatorname{Hom}_{S_{2}}\left(-, S_{2} M\right)$, and therefore, by Proposition 3.1, the functor $\operatorname{Hom}_{S}(-, M)$ is e-injective as soon as the functors $\operatorname{Hom}_{S_{1}}\left(-, S_{1} M\right)$ and $\operatorname{Hom}_{S_{2}}\left(-, S_{2} M\right)$ are einjective as well. Now it follows immediately that $S \in \mathfrak{C}(\mathfrak{F})$ if we observe that the semimodules $S_{1} M$ and $S_{2} M$ are cyclic, or finitely generated, provided that the semimodule $M$ is itself cyclic, or finitely generated, respectively.

Proposition 6.2 The following conditions for a semiring $S$ are equivalent:

(1) All cyclic (finitely generated) left S-semimodules are e-injective; 
(2) $S \simeq R \oplus T$, where $R$ is a (classical) semisimple ring, and $T$ is a zerosumfree semiring with an infinite element whose all cyclic (finitely generated) left semimodules are e-injective.

Proof $(1) \Longrightarrow(2)$. Let $\equiv_{V(S)}$ be the Bourne congruence on $S$. It is clear that the factor semiring $\bar{S}:=S / \equiv_{V(S)}$ is zerosumfree. So, by Proposition 6.1, every cyclic (finitely generated) left $\bar{S}$-semimodule is e-injective. In particular, $\bar{S} \bar{S}$ is e-injective and, by Proposition 4.1, contains an infinite element whence a zeroic zerosumfree semiring. Whence, by [18, Proposition 2.9], $S=R \oplus T$, where $R$ is a ring and $T$ is a semiring isomorphic to $\bar{S}$. Since $R$ is a homomorphic image of $S$, all cyclic (finitely generated) left $R$-modules are injective by Proposition 6.1, and hence, by the celebrated Osofsky's Theorem ([41, Theorem, p. 649]) $R$ is a semisimple ring.

$(2) \Longrightarrow(1)$. This follows immediately from Proposition 6.1 and [41, Theorem; p. 649].

As was shown in [16], all finitely generated left $S$-semimodules over a semiring $S$ are injective if and only if a semiring $S$ is a (classical) semisimple ring. However, as we will demonstrate below, in the general semiring setting the e-injective version of this result is not true. From Proposition 6.2, we see that the problem of describing semirings all of whose cyclic (finitely generated) semimodules are e- injective is actually reduced to the corresponding problem for the class of zerosumfree semirings with infinite elements that, particularly, includes the very important subclass of bounded distributive lattices. Therefore, it is quite natural that a characterization of bounded distributive lattices all of whose cyclic (finitely generated) semimodules are e-injective constitutes our next goal, to achieve which we need first to establish the following important facts.

Lemma 6.3 If $S$ is a bounded distributive lattice all of whose cyclic semimodules are e-injective, then it is a Boolean algebra.

Proof First let us show that for any $a \in S$, we have $S a+A n n(a)=S$, where $A n n(a):=(0: a)$ is the annihilator of the element $a$. Indeed, suppose that $S a+$ $\operatorname{Ann}(a) \varsubsetneqq S$, then $S a+A n n(a)$ is contained in a maximal ideal $I$ of $S$. Obviously, $I$, as any ideal of any bounded distributive lattice, is strongly subtractive. It implies that the set $\{0\} \cup\{s a \mid s \in S \backslash I\}$ is a submonoid of the monoid $(S,+, 0)$. Moreover, by [14, Corollary 7.13], $I$ is also a prime ideal of $S$. If $s a \neq 0$ for all $s \in S \backslash I$, then the monoid $\{0\} \cup\{s a \mid s \in S \backslash I\}$ becomes a left $S$-semimodule by setting $s(r a)=0$ for all $s \in I$ and $r \notin I$, and $s(r a)=s r a$ for all $s \notin I$ and $r \notin I$. Next let us take an arbitrary element $m \notin S$ and on the set $V:=\{0\} \cup\{s a \mid s \in$ $S \backslash I\} \cup\{m\}$ extend the operations of the $S$-semimodule $\{0\} \cup\{s a \mid s \in S \backslash I\}$ by setting $m+0=0+m=m+m=m, m+s a=s a+m=s a$ for all $s \in S \backslash I$, and $s m=0$ for all $s \in I$, and $s m=m$ for all $s \in S \backslash I$. One can easily verify that $V$ becomes a left $S$-semimodule. Obviously, $M:=\{0, m\}$ is a 
subtractive $S$-subsemimodule of $V$. Clearly, $M$ is cyclic and, hence, e-injective; therefore, there exists an $S$-homomorphism $f: V \longrightarrow M$ such that $\left.f\right|_{M}=i d_{M}$. It implies that $f(s a)=m$ for all $s \in S \backslash I$. In particular, we have that $f(a)=m$. On the other hand, since $\operatorname{Sa}+\operatorname{Ann}(a) \subseteq I$, we get that $a \in I$, and hence, $f(a)=f\left(a^{2}\right)=a f(a)=a m=0$. Whence, $s a=0$ for some $s \in S \backslash I$, that is, $s \in \operatorname{Ann}(a) \subseteq I$, and hence, $s \in I$; and therefore, this contradiction implies $S=S a+\operatorname{Ann}(a)$ for all $a \in S$.

Now we show that $S$ is a Boolean algebra. Indeed, for any $a \in S$, we have $S=S a+\operatorname{Ann}(a)$; and hence, $1=s a+x$ for some $s \in S$ and $x \in \operatorname{Ann}(a)$. We then have that

$$
1=a+1=a+s a+x=a(1+s)+x=a 1+x=a+x .
$$

For $x \in \operatorname{Ann}(a)$, we have $x a=0$, and therefore, $x$ is the complement to $a$, and $S$ is a Boolean algebra.

Moreover, for the semiring $S$ in the previous lemma, we can make a more precise observation, namely:

Lemma 6.4 If $S$ is a bounded distributive lattice all of whose cyclic semimodules are e-injective, then $S$ is a complete Boolean algebra.

Proof By [6, Theorem X.9] (or, [45, Theorem 8.5]) and Lemma 6.3, the Boolean algebra $S$ can be considered as a Boolean subalgebra of a complete Boolean algebra $B$; and hence, $S \subseteq B, 0_{S}=0_{B}$, and $1_{S}=1_{B}$, and $B$ is naturally a left $S$-semimodule. Define on the left $S$-semimodule $S \times B$ the relation $\sim$ as follows: $\left(s_{1}, b_{1}\right) \sim\left(s_{2}, b_{2}\right)$ iff 1) $b_{1}=b_{2}$ and 2) there exist $n \in \mathbb{N}, x_{i}, x_{i}^{\prime} \in B, s_{i}^{\prime}, s_{i}^{\prime \prime} \in S$, $i=1, \ldots, n$, such that $x_{i}+x_{i}^{\prime}=b_{1}$ for all $i$, and $s_{1}+\sum_{i=1}^{n} s_{i}^{\prime} x_{i}=s_{2}+\sum_{i=1}^{n} s_{i}^{\prime \prime} x_{i}$. Repeating verbatim the proof of [17, Proposition 1.6], one easily sees that $\sim$ is a congruence on ${ }_{S}(S \times B)$.

Let $\widetilde{S}:=(S \times B) / \sim$ and $[s, b]$ be the class containing the pair $(s, b)$. It is easy to see that the image of the embedding $\mu: S \longmapsto \widetilde{S}, s \longmapsto[s, 0]$, is a subtractive $S$ semimodule of ${ }_{S} \widetilde{S}$. Hence, for ${ }_{S} S$ is e-injective, there exists an $S$-homomorphism $\varphi: \widetilde{S} \rightarrow S$ such that $\varphi \mu=1_{S}$. By the dual of [6, Theorem IX.2], in order to prove $S$ is complete, it is sufficient to show that every family $\left\{s_{i}\right\}_{i \in I}$ of elements in $S$ has a least upper bound $s \in S$. Since $S \subseteq B$ and $B$ is complete, a family $\left\{s_{i}\right\}_{i \in I}$ has a least upper bound $b \in B$ in $B$, and let $s:=\varphi([0, b])$. For each $i \in I$, it is easy to see that $\left[s_{i}, b\right]=[0, b]$ : Indeed, $\left(s_{i}, b\right) \sim(0, b)$ by putting $n=1, x_{1}=b$, $x_{1}^{\prime}=0, s_{1}^{\prime}=0, s_{1}^{\prime \prime}=s_{i}$. Therefore,

$$
s_{i}+s=\varphi\left(\mu\left(s_{i}\right)\right)+\varphi([0, b])=\varphi\left(\left[s_{i}, 0\right]+[0, b]\right)=\varphi\left(\left[s_{i}, b\right]\right)=\varphi([0, b])=s,
$$

and hence, $s$ is an upper bound for $\left\{s_{i}\right\}_{i \in I}$ in $S$. If $y \in S$ is another upper bound for $\left\{s_{i}\right\}_{i \in I}$, then $b \leq y$ as $b$ is the least upper bound for $\left\{s_{i}\right\}_{i \in I}$ in $B$; and 
therefore, $b y=b$ and $s y=\varphi([0, b]) y=\varphi([0, b y])=\varphi[0, b]=s$, i.e., $s \leq y$ and $s$ is a least upper bound for $\left\{s_{i}\right\}_{i \in I}$ in $S$.

Now we are ready to characterize bounded distributive lattices all of whose cyclic (finitely generated) semimodules are e-injective, namely:

Theorem 6.5 The following conditions for a bounded distributive lattice $S$ are equivalent:

(1) All finitely generated $S$-semimodules are e-injective;

(2) All cyclic S-semimodules are e-injective;

(3) $S$ is a finite Boolean algebra.

Proof (1) $\Longrightarrow(2)$. It is obvious.

$(2) \Longrightarrow(3)$. By Lemma 6.4, $S$ is a complete Boolean algebra. Suppose that $S$ is infinite, then, by [3, Lemma 4.2] for example, it contains a countable set of orthogonal idempotents $\left\{e_{n} \mid n \in \mathbb{N}\right\}$, and we have the ideal $I:=\Sigma_{n \in \mathbb{N}} S e_{n}$ and the factor algebra $\bar{S}:=S / I$. For $\bar{S}$ is a Boolean algebra and by Proposition 6.1, we have that all cyclic $\bar{S}$-semimodules are e-injective and, by Lemma 6.4 again, $\bar{S}$ is a complete Boolean algebra. On the other hand, repeating verbatim the proof of [3, Theorem 4.3], we have that $\bar{S}$ is not a complete Boolean algebra, and from this contradiction we conclude that $S$ is a finite Boolean algebra.

$(3) \Longrightarrow(1)$. Let $S$ be a finite Boolean algebra and $\left\{e_{1}, e_{2}, \ldots, e_{n}\right\}$ the set of all atoms of $S$. Clearly, $S=S e_{1} \oplus S e_{2} \oplus \ldots \oplus S e_{n}$ and $S e_{i}=\left\{0, e_{i}\right\} \simeq \mathbf{B}$ for each $i$. Then, applying Theorem 4.4 (5) and Proposition 6.1, we conclude the proof.

As a corollary of Theorem 6.5, we are able to extend the characterization of finite Boolean algebras among bounded distributive lattices given in [3, Corollary 4.4]:

Corollary 6.6 The following conditions for a bounded distributive lattice $S$ are equivalent:

(1) All cyclic S-semimodules are projective;

(2) All subsemimodules of the regular semimodule ${ }_{S} S$ are injective;

(3) All cyclic S-semimodules are e-injective;

(4) All cyclic $S$-semimodules are injective;

(5) $S$ is a finite Boolean algebra.

As another consequence of Theorem 6.5, we obtain a complete description of subtractive semirings all of whose finitely generated semimodules are e-injective.

Theorem 6.7 The following conditions for a left subtractive semiring $S$ are equivalent:

(1) All finitely generated S-semimodules are e-injective; 
(2) All cyclic $S$-semimodules are e-injective;

(3) $S \simeq R \oplus T$, where $R$ is a semisimple ring and $T$ is a finite Boolean algebra. Proof $(1) \Longrightarrow(2)$. It is obvious.

$(2) \Longrightarrow(3)$. By Proposition 6.2, $S \simeq R \oplus T$, where $R$ is a semisimple ring and $T$ a semiring with an infinite element $\infty$ such that all cyclic left $T$-semimodules are e-injective. By [25, Lemma 4.7], $T$ is a left subtractive semiring. Whence, the left ideal $T \infty$ is a subtractive ideal of $T$ and it follows from $1_{T}+\infty=\infty$ that $1_{T} \in T \infty$, i.e., $t \infty=1_{T}$ for some $t \in T$. From the latter and $\infty^{2}+\infty=\infty$, we have $\infty=\infty+1_{T}=1_{T}$, and hence, $x+1_{T}=1_{T}$ for all $x \in T$.

As, for each $a \in T$, the cyclic left $T$-semimodule $T a$ is both e-injective and subtractive subsemimodule of $T$ by our hypothesis. Therefore, there exists a homomorphism $\varphi: T \longrightarrow T a$ such that $\left.\varphi\right|_{T a}=1_{T a}$. In particular, $a=\varphi(a)=$ $\varphi(a 1)=a \varphi(1)$ and $\varphi(1) \in T a$. Hence, $\varphi(1)=x a$ for some $x \in T$ and $a=a x a$. From the latter, repeating verbatim the proof of [3, Theorem 4.6], we immediately obtain that $T$ is a bounded distributive lattice, and therefore, applying Theorem 6.5 , we conclude that $T$ is a finite Boolean algebra.

$(3) \Longrightarrow(1)$. This follows immediately from Proposition 6.1, Theorem 6.5, and [41, Theorem, p. 649].

By [19, Corollary 3.2], every $S$-semimodule over a semiring $S$ can be canonically represented as a colimit of a diagram of cyclic $S$-semimodules. In light of this observation, Corollary 6.6 and Theorem 6.7, it seems to be reasonable and interesting the following problem.

Problem 5 Describe all semirings $S$ for which the classes of cyclic injective and cyclic e-injective semimodules coincide.

Now, in what follows, we use the concepts and notations from 2.4, 2.5 and 2.6, and following [2, we say that a right $S$-semimodule $P_{S}$ is $e$-flat iff the functor $P \otimes_{S}-:{ }_{S} \mathcal{M} \longrightarrow \mathcal{M}$ preserves short exact sequences or equivalently iff the functor $P \otimes_{S}-$ preserves subtractive subsemimodules.

Lemma 6.8 Every projective right $S$-semimodule is e-flat.

Proof As was shown in [22] and [24], any tensor product functor $P \otimes_{S}-$ : ${ }_{S} \mathcal{M} \longrightarrow \mathcal{M}$ has a right adjoint, and therefore, by [38, The dual Theorem 5.5.1], preserves colimits, in particular, coproducts. Then, for projective semimodules are retracts of free ones, the statement follows right away from [22, Proposition 3.8] and Corollary 3.3.

Lemma 6.9 Let $F:{ }_{S} \mathcal{M} \rightleftarrows{ }_{T} \mathcal{M}: G$ be an equivalence between the semimodule categories ${ }_{S} \mathcal{M}$ and ${ }_{T} \mathcal{M}$. Then a left $S$-semimodule ${ }_{S} M \in{ }_{S} \mathcal{M}$ is e-injective iff ${ }_{T} F(M) \in{ }_{T} \mathcal{M}$ is e-injective.

Proof By [26, Theorems 4.5 and 4.12], $F \simeq P \otimes_{S}-$ for some $(T, S)$-bisemimodule $P$ such that $P_{S}$ is a progenerator, $P^{*}:=\operatorname{Hom}_{S}\left(P_{S}, S_{S}\right)$ is a progenerator in $\mathcal{M}_{T}$ 
and $T \simeq \operatorname{End}\left(P_{S}\right)$ as semirings, and $G \simeq P^{*} \otimes_{T}-$. Since projective semimodules are e-flat by Lemma 6.8 , both $F$ and $G$ preserve short exact sequences, and the statement readily follows from the natural functor isomorphisms $F G \simeq I d_{S} \mathcal{M}$ and $G F \simeq I d_{T \mathcal{M}}$ [38, Sect. 4.4] (see also the dual of [26, Lemma 4.10]).

We conclude these section and paper by giving a complete characterization of simple semirings all of whose finitely generated left semimodules are e-injective.

Theorem 6.10 The following conditions for a semiring $S$ are equivalent:

(1) $S$ is a simple semiring all of whose finitely generated left semimodules are e-injective;

(2) $S$ is a simple semiring all of whose cyclic left semimodules are e-injective;

(3) $S$ is isomorphic either to a matrix semiring $M_{n}(D)$ for some division ring $D$ and $n \geq 1$, or to an endomorphism semiring End $(L)$ of a nonzero finite distributive lattice $L$.

Proof $(1) \Longrightarrow(2)$. It is obvious.

$(2) \Longrightarrow(3)$. By Proposition 6.2, $S$ is a simple semisimple ring, or $S$ is a simple semiring with an infinite element. If $S$ is a simple semisimple ring, then $S \simeq M_{n}(D)$ for some division ring $D$ and $n \geq 1$ by the classical WedderburnArtin structure theorem for rings (see, e.g., [36, Wedderburn-Artin Theorem 3.5]). Otherwise, $S$ is a simple semiring with an infinite element $\infty$. In particular, $\infty+\infty=\infty$, whence $\infty \in I^{+}(S)$ and hence $I^{+}(S)$ is a nonzero ideal of $S$. For $S$ is a simple semiring, $I^{+}(S)=S$ and $S$ is an additively idempotent simple semiring containing an infinite element, and therefore, by [28, Theorem 5.7], $S \simeq \operatorname{End}(L)$ for some nonzero finite distributive lattice $L$.

$(3) \Longrightarrow(1)$. Case I: Let $S \simeq M_{n}(D)$ for some division $\operatorname{ring} D$ and $n \geq 1$. It follows by the Wedderburn-Artin Theorem and the celebrated Osofsky's result [41, Theorem], all finitely generated left $S$-semimodules are (e-)injective.

Case II: Let $S \simeq \operatorname{End}(L)$ for some nonzero finite distributive lattice $L$. Then, by [28, Theorem 5.7], $S$ is a simple semiring that Morita equivalent to the Boolean semiring $\mathbf{B}$; and let the functors $F:{ }_{S} \mathcal{M} \rightleftarrows{ }_{\mathbf{B}} \mathcal{M}: G$ establish an equivalence between the semimodule categories ${ }_{S} \mathcal{M}$ and ${ }_{\mathrm{B}} \mathcal{M}$. For any finitely generated left $S$-semimodule $\left.M \in\right|_{S} \mathcal{M} \mid$, by [26, Proposition 4.8] and Theorem 4.4 (5), the semimodule $\left.F(M) \in\right|_{\mathbf{B}} \mathcal{M} \mid$ is a finitely generated e-injective $\mathbf{B}$-semimodule. Then, applying Lemma 6.9 and the natural isomorphism $M \simeq G(F(M))$, we have that the semimodule $M \in{ }_{S} \mathcal{M} \mid$ is e-injective as well and end the proof.

Acknowledgement: The first and fourth authors would like to acknowledge a support provided by the Deanship of Scientific Research (DSR) at King Fahd University of Petroleum \& Minerals (KFUPM) for funding this work through project RG1304. 


\section{References}

[1] J. Abuhlail, Exact sequences of commutative monoids and semimodules, Homology Homotopy Appl. 16 (1) (2014), 199-214.

[2] J. Abuhlail, Some remarks on tensor products and flatness of semimodules, Semigroup Forum 88 (3) (2014), 732-738.

[3] J. Y. Abuhlail, S. N. Il'in, Y. Katsov, T. G. Nam, On $V$-Semirings and Semirings all of whose Cyclic Semimodules are Injective, Communications in Algebra, 43 (2015), 4632-4654.

[4] F. W. Anderson and K. R. Fuller, Rings and Categories of Modules, 2nd ed., Springer-Verlag, New York-Berlin, 1979.

[5] R. El Bashir, J. Hurt, A. Jančařík, and T. Kepka, Simple Commutative Semirings, J. Algebra, 236 (2001), 277-306.

[6] G. Birkhoff, Lattice Theory, American Mathematical Society, Providence, 1967.

[7] F. Borceux, Handbook of Categorical Algebra. I, Basic Category Theory, Cambridge Univ. Press (1994).

[8] A. Connes and C. Consani, Schemes over $\mathbb{F}_{1}$ and zeta functions, Compos. Math., 146 (2010), 1383-1415.

[9] J. Giansiracusa and N. Giansiracusa, Equations of tropical varieties, to appear in Duke Mathematical Journal (see also preprint: arXiv:1308.0042v2).

[10] T. S. Fofanova, Injectivity of polygons over Boolean algebras, Siberian Math. J., 13 (1972), 452-458 (in Russian).

[11] T. S. Fofanova, Polygons over distributive lattices, in: Universal Algebra, Colloq. Math. Soc. János Bolyai \# 29, North-Holland Publishing Co., Amsterdam, 1982, 289-292.

[12] S. I. Gelfand, Y. I. Manin, Methods of homological algebra, Second edition, Springer Monographs in Mathematics. Springer-Verlag, Berlin, 2003

[13] K. Głazek(2001). A Guide to the Literature on Semirings and their Applications in Mathematics and Information Science, Kluwer Academic Publishers, Dordrecht-Boston-London, 2001

[14] J. S. Golan, Semirings and their Applications, Kluwer Academic Publishers, Dordrecht-Boston-London, 1999. 
[15] A. Horn, N. Kimura, The category of semilattices, Algebra Universalis, 1 (1971), $26-38$.

[16] S. N. Il'in, Semirings over Which all Semimodules are Injective (Projective), Matem. Vestn. Pedvuzov i Univ. Volgo-Vyatsk. Regiona, 8 (2006), 50-53.

[17] S. N. Il'in, Direct Sums of Injective Semimodules and Direct Products of Projective Semimodules Over Semirings, Russian Mathematics, 54 (2010), 27 - 37.

[18] S. N. Il'in, V-semirings, Siberian Mathematical Journal, 53 (2012), 222 - 231.

[19] S. N. Il'in, Y. Katsov, T.G. Nam, Toward Homological Structure Theory of Semimodules: On Semirings All of Whose Cyclic Semimodules Are Projective, preprint: arXiv:1509.02997v1.

[20] Z. Izhakian, L. Rowen, Supertropical algebra, Adv. Math., 225(4) (2010), 22222286 .

[21] J. Jun, C̆ech cohomology of semiring schemes, preprint: arXiv:1503.01389v1.

[22] Y. Katsov, Tensor products and injective envelopes of semimodules over additively regular semirings, Algebra Colloquium, 4 (1997), 121-131.

[23] Y. Katsov, On flat semimodules over semirings, Algebra Universalis, 51 (2004), 287-299.

[24] Y. Katsov, Toward homological characterization of semirings: Serre's conjecture and Bass's perfectness in a semiring context, Algebra Universalis, 52 (2004), 197214 .

[25] Y. Katsov, T. G. Nam, N. X. Tuyen, On subtractive semisimple semirings, Algebra Colloquium, 16 (2009), 415-426.

[26] Y. Katsov and T. G. Nam, Morita Equivalence and Homological Characterization of Semirings, J. Algebra Appl., 10 (2011), 445 - 473.

[27] Y. Katsov, T. G. Nam, N. X. Tuyen, More on Subtractive Semirings: Simpleness, Perfectness and Related Problems, Comm. Algebra, 39 (2011), 4342 - 4356.

[28] Y. Katsov, T. G. Nam, J. Zumbrägel, On Simpleness of Semirings and Complete Semirings, J. Algebra Appl., 13: 6 (2014). DOI: 10.1142/S0219498814500157.

[29] Y. Katsov, T. G. Nam, On radicals of semirings and related problems, Comm. Algebra 42 (2014), no. 12, 5065-5099. 
[30] Y. Katsov, T. G. Nam, J. Zumbrägel, Simpleness of Leavitt path algebras with coefficients in commutative semiring, Semigroup Forum, 2016. DOI 10.1007/s00233-016-9781-1.

[31] A. Kendziorra and J. Zumbrägel, Finite simple additively idempotent semirings, J. Algebra, 388 (2013), 43-64.

[32] T. Kepka, J. Kortelainen and P. Nĕmec, Simple semirings with zero, J. Algebra Appl.. 15: 3 (2016) 1650047. DOI: 10.1142/S021949881650047X.

[33] T. Kepka and P. Nĕmec, Simple semirings with left muliplicatively absorbing elements, Semigroup Forum, 91 (2015), 159-170.

[34] M. Kilp, U. Knauer, A. V. Mikhalev, Monoids, Acts and Categories, Walter de Gruyter, Berlin-New York, 2000.

[35] T. Y. Lam, Lectures on Modules and Rings, Springer-Verlag, New York-Berlin, 1999.

[36] T. Y. Lam, A first course in noncommutative rings, 2nd Ed., Springer-Verlag, New York-Berlin, 2001.

[37] O. Lorscheid, The geometry of blueprints. Part I: Algebraic background and scheme theory, Adv. Math., 229(3) (2012), 1804-1846.

[38] S. Mac Lane, Categories for the Working Mathematician, Springer-Verlag, New York-Berlin, 1971.

[39] G. Maze, C. Monico, and J. Rosenthal, Public key cryptography based on semigroup actions, Adv. Math. Commun., 1 (2007), 489-507.

[40] C. Monico, On finite congruence-simple semirings, J. Algebra, 271 (2004), 846854.

[41] B. L. Osofsky, Rings all of whose finitely generated modules are injective, Pacific J. Math., 14 (1964), 645 - 650.

[42] A. Patchkoria, Extensions of Semimodules and Takahashi functor $\operatorname{Ext}_{\Lambda}(C, A)$, Homology, Homotopy and Appl., 5 (2013), 387 - 406.

[43] J. Richter-Gebert, B. Sturmfels, T. Theobald, First steps in tropical geometry, in: Idempotent Mathematics and Mathematical Physics, in: Contemp. Math., vol. 377, American Mathematical Society, Providence, RI, 2005, pp. 289-317.

[44] H. Schubert, Categories, Springer-Verlag, New York-Heidelberg, 1972. 
[45] L. A. Skornyakov, L. A. Elementy teorii struktur (Russian) [Elements of lattice theory], Second edition, "Nauka", Moscow, 1982.

[46] M. Takahashi, On the bordism categories. II. Elementary properties of semimodules, Kobe J. Math., 9 (1981), 495-530.

[47] M. Takahashi, On the bordism categories. III. Functors Hom and for semimodules, Math. Sem. Notes Kobe Univ. 10 (2) (1982), 551-562.

[48] M. Takahashi, Completeness and c-cocompleteness of the category of semimodules, Kobe J. Math., 10 (1982), 551-562.

[49] M. Takahashi, Extensions of semimodules I, Kobe J. Math., 10 (1982), 563-592.

[50] J. Zumbrägel, Classification of finite congruence-simple semirings with zero, $J$. Algebra Appl., 7 (2008), 363-377. 